\title{
Next-Generation Sequencing and In Silico Analysis Facilitate Prolonged Response to Pazopanib in a Patient With Metastatic Urothelial Carcinoma of the Renal Pelvis
}

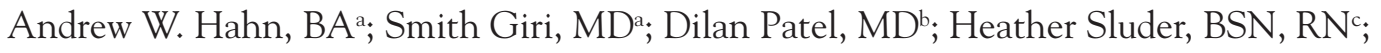 \\ Ari Vanderwalde, MD, MPH ${ }^{\mathrm{a}, \mathrm{c}}$; and Mike G. Martin, MD ${ }^{\mathrm{a}, \mathrm{c}}$
}

\begin{abstract}
With the advent of widespread tumor genetic profiling, an increased number of mutations with unknown significance are being identified. Often, a glut of uninterpretable findings may confuse the clinician and provide little or inappropriate guidance in therapeutic decisionmaking. This report describes a method of protein modeling by in silico analysis (ie, using computer simulation) that is easily accessible to the practicing clinician without need for further laboratory analysis, which can potentially serve as a guide in therapeutic decisions based on poorly characterized tumor mutations. An example of this model is given wherein poorly characterized KIT, PDGFRB, and ERBB2 mutations were discovered in a patient with treatment-refractory metastatic transitional cell carcinoma of the renal pelvis. The $K I T$ and $P D G F R B$ mutations were predicted to be pathogenic using in silico analysis, whereas the ERBB2 mutation was predicted to be benign. Based on these findings, the patient was treated with pazopanib and achieved a partial response that lasted for 7.5 months. We propose that in silico analysis be explored as a potential means to further characterize genetic abnormalities found by tumor profiling assays, such as nextgeneration sequencing. (J Natl Compr Canc Netw 2015;13:1181-1185)
\end{abstract}

Urothelial carcinoma of the renal pelvis (UCRP) and the ureter is a rare disease, representing $5 \%$ of all urothelial malignancies. The incidence of UCRP in the general US population is estimated to be 1.15 per 100,000 person-years. ${ }^{1}$ Although radical nephroureterectomy is the therapy of choice for nonmetastatic UCRP, the rate of tumor recurrence is still high. For metastatic UCRP (mUCRP), cisplatin-based chemotherapies are often used as first-line agents with limited response, largely based

From the a Department of Internal Medicine, The University of Tennessee Health Science Center, Memphis, Tennessee; ${ }^{b}$ Department of Internal Medicine, Washington University in St. Louis, St. Louis, Missouri; and 'Department of Hematology/ Oncology, The West Cancer Center, Memphis, Tennessee. Submitted November 18, 2014; accepted for publication May 21, 2015.

The authors have disclosed that they have no financial interests, arrangements, affiliations, or commercial interests with the manufacturers of any products discussed in this article or their competitors.

Correspondence: Andrew W. Hahn, BA, The University of Tennessee Health Science Center/West Cancer Center, 1588 Union Avenue, c/o Dr. Mike G. Martin, Memphis, TN 38104. E-mail: ahahn1@uthsc.edu on experience from urothelial tumors of the bladder. ${ }^{2}$ However, second-line chemotherapies for urothelial malignancies have significantly lower response rates and no significant survival benefit over supportive care. ${ }^{2}$

Whole-genome studies of metastatic urothelial cancer have shown profound genomic heterogeneity at the nucleotide and chromosomal levels. ${ }^{3,4}$ Furthermore, the mutation profiles of histologically similar tumors may vary widely, explaining the variable response to chemotherapy observed in small clinical trials. ${ }^{5}$ The availability of next-generation sequencing (NGS) has made it feasible for clinicians to analyze genetic profiles of an individual's cancer. When these results are coupled with the continual development of targeted therapies, personalized medicine is possible. However, interpretation of the numerous genetic variants that NGS provides and selection of an appropriate targeted therapy presents a novel challenge to clinicians. This report documents the successful use of NGS coupled with in silico analysis to select a targeted therapy, pazopanib, from nu- 
merous potential mutations in a patient with heavily pretreated mUCRP.

\section{Case Report}

A 61-year-old woman presented to our clinic in November 2010 with intermittent hematuria for 2 months. Further evaluation revealed a left highgrade UCRP, and the patient underwent a radical nephroureterectomy (pT2NXMO). The patient received 3 cycles of adjuvant gemcitabine plus cisplatin (GC) but relapsed with biopsy-proven urothelial pulmonary metastases. Subsequently, the patient received sequential chemotherapies, including 6 months of palliative carboplatin and gemcitabine with a partial response (PR), 4 months of pemetrexed with a PR, 5 months of paclitaxel with stable disease (SD), and 2 months of methotrexate with progressive disease (PD). Each chemotherapy change was mandated by PD on CT. Her performance status remained excellent despite her PD and extensive chemotherapy exposure.

Because of a lack of validated therapeutic options, a PrecipioNGS-421 whole-exome sequencing (WES) analysis (Yale School of Medicine, New Haven, CT) was conducted on the biopsied metastatic tissue that revealed potentially targetable mutations in ERBB2 (UniProtKB \#P04626), KIT (\#P10721), and PDGFRB (\#P09619), and other genes (Table 1). Precipio uses DNA extracted from tumor cells with custom HaloPlex reagents and is sequenced on the Ion Torrent Personal Genome Machine (Illumina platform). ${ }^{6,7}$ The significance of the mutations was then predicted in silico using Harvard's PolyPhen-2 $(1,2,3)$ algorithm on a personal computer. PolyPhen-2 is a "tool which predicts possible impact of an amino acid substitution on the structure and function of a human protein using straightforward physical and comparative considerations." ${ }^{8}$ PolyPhen-2 uses "protein sequences from UniProtKB/UniRef100 Release 2011_12..., structures from PDB/DSSP Snapshot 03-Jan-2012... and UCSC MultiZ multiple alignments of 45 vertebrate genomes with hg19/ GRCh37 human genome..." to make predictions. ${ }^{8}$ The KIT mutation had not been previously described or targeted in UCRP but is sensitive to pazopanib in other malignancies. ${ }^{9}$ PolyPhen-2 predicted the mutations in KIT and PDGFRB to be pathogenic and the ERBB2 mutation to be benign. Our patient was started on off-label pazopanib, which has activity against KIT and PDGFRB mutations, and remained on therapy for more than 7.5 months with minimal side effects and stabilization of her previous weight loss and her CT scan findings (SD in the bones and liver and a 50\% reduction in lung metastases). Her response to pazopanib was her longest response to any agent. After progression, her performance status deteriorated and she elected hospice care.

\section{Discussion}

mUCRP has a grim prognosis, with a 3-year survival rate of less than $10 \% .^{1}$ First-line chemotherapy for mUCRP constitutes GC or methotrexate, vinblastine, doxorubicin, and cisplatin (MVAC). ${ }^{2,10}$ Clinical trials among patients with all urothelial malignancies have

\section{Table 1 Selected Mutations Found in Patients With mUCRP Using Next-Generation Sequencing}

\begin{tabular}{|c|c|c|c|c|c|}
\hline Gene & Variant & Effect & Clinical Significance $^{a}$ & PolyPhen-2 Score & Prediction \\
\hline KIT & $\begin{array}{l}\text { c. } 2662 C>T \\
\text { p.R888W }\end{array}$ & $\begin{array}{l}\text { Point } \\
\text { mutation }\end{array}$ & $\begin{array}{l}\text { Previously described in other } \\
\text { malignancies and associated with } \\
\text { clinical responses }\end{array}$ & $\begin{array}{l}1.000 \text { (sensitivity, } 0.00 \\
\text { specificity, 1.00) }\end{array}$ & $\begin{array}{l}\text { Probably } \\
\text { damaging }\end{array}$ \\
\hline PDGFRB & $\begin{array}{l}\text { c. } 595 \mathrm{G}>\mathrm{A} \\
\text { p.V199M }\end{array}$ & $\begin{array}{l}\text { Point } \\
\text { mutation }\end{array}$ & $\begin{array}{l}\text { Not previously described, clinical } \\
\text { trials are open }\end{array}$ & $\begin{array}{l}0.997 \text { (sensitivity, } 0.41 \\
\text { specificity, 0.98) }\end{array}$ & $\begin{array}{l}\text { Probably } \\
\text { damaging }\end{array}$ \\
\hline PDGFRB & $\begin{array}{l}\text { c.596T>G } \\
\text { p.V199G }\end{array}$ & $\begin{array}{l}\text { Point } \\
\text { mutation }\end{array}$ & $\begin{array}{l}\text { Not previously described, clinical } \\
\text { trials are open }\end{array}$ & $\begin{array}{l}0.988 \text { (sensitivity, } 0.73 \\
\text { specificity, } 0.96 \text { ) }\end{array}$ & $\begin{array}{l}\text { Probably } \\
\text { damaging }\end{array}$ \\
\hline ERBB2 & $\begin{array}{l}\text { c.3557C }>A \\
\text { p.A1186D }\end{array}$ & $\begin{array}{l}\text { Point } \\
\text { mutation }\end{array}$ & $\begin{array}{l}\text { Unknown functional significance, } \\
\text { clinical trials are open }\end{array}$ & $\begin{array}{l}0.049 \text { (sensitivity, 0.94; } \\
\text { specificity, 0.83) }\end{array}$ & Benign \\
\hline CHEK1 & $\begin{array}{l}\text { c. } 749 C>A \\
\text { p.P250Q }\end{array}$ & $\begin{array}{l}\text { Point } \\
\text { mutation }\end{array}$ & $\begin{array}{l}\text { Unknown functional significance, } \\
\text { clinical trials are open }\end{array}$ & $\begin{array}{l}1.000 \text { (sensitivity, } 0.00 \\
\text { specificity, } 1.00 \text { ) }\end{array}$ & $\begin{array}{l}\text { Probably } \\
\text { damaging }\end{array}$ \\
\hline PIK3CG & $\begin{array}{l}\text { c. } 2191 \mathrm{G}>\mathrm{A} \\
\text { p.D731N }\end{array}$ & $\begin{array}{l}\text { Point } \\
\text { mutation }\end{array}$ & $\begin{array}{l}\text { Unknown functional significance, } \\
\text { clinical trials are open }\end{array}$ & $\begin{array}{l}0.831 \text { (sensitivity, } 0.84 ; \\
\text { specificity, } 0.93 \text { ) }\end{array}$ & $\begin{array}{l}\text { Possibly } \\
\text { damaging }\end{array}$ \\
\hline
\end{tabular}

aAs reported by Precipio.

Abbreviation: mUCRP, metastatic urothelial carcinoma of the renal pelvis. 
shown response rates ranging from $40 \%$ to $72 \%$ for GC and MVAC, but no trials have specifically examined the response rates among patients with UCRP. ${ }^{11,12}$ Doserelated and cumulative renal toxicities are the most common dose-limiting adverse effects in the treatment of UCRP. Among the first-line agents, cisplatin and methotrexate are nephrotoxic agents that are renally excreted. Because most patients with UCRP are elderly and many have had prior radical nephroureterectomy, the concern for renal toxicity frequently limits the use of these chemotherapeutic regimens. ${ }^{11}$

The poor outcomes and significant toxicities associated with first-line treatment of mUCRP create a need for novel second-line therapies, yet the rarity of the disease has limited guidance from clinical trials. The paucity of meaningful trials has created an opportunity for NGS to provide clinicians with meaningful data for the personalized treatment of heavily pretreated metastatic disease. However, the wealth of information provided by NGS presents clinicians with a new challenge in deciphering which genomic alterations should be acted upon. For example, Ross et $\mathrm{al}^{13}$ recently published the results of genomic profiling in 125 patients with cancer of unknown primary, and found a mean of 4 genetic alternations per patient. This case presents an example of not only how NGS can produce a wealth of potentially useful data but also how in silico analysis of the results of NGS can help guide physicians in selecting appropriate, off-label targeted therapy.

NGS technologies were first commercially introduced in 2007.14 NGS platforms, such as Illumina or Ion Torrent, have increased the sensitivity and scalability for DNA sequencing of cancer cells to provide practical genetic information. ${ }^{15}$ An array of NGS applications exist, including DNA sequencing, which can be used for whole-genome sequencing, WES, or sequencing of specific targeted "hot spots" of the genome. ${ }^{14}$ WES is most useful in clinical diagnostics because it analyzes exons, which only account for approximately $2 \%$ of all genomic DNA but still encompass the DNA relevant to targeted therapies. We used Precipio (one of the many companies that offer NGS) WES of 421 genes to yield our initial NGS results. ${ }^{16}$ Precipio uses the Illumina and Ion Torrent NGS platforms to produce its WES results. WES results provide data on point mutations present within exons of the tissue specimen provided. Precipio, like most NGS services available, provides only the mutations present and advises whether these mutations are the focus of any targeted therapies or current clinical trials. The challenge that oncologists face is deciphering which mutations of the many listed by NGS are driving a particular malignancy and should be the focus of targeted therapy. For example, our patient's NGS revealed mutations in ERBB2, which is seen in 9\% of urothelial carcinomas (UCs), and in KIT and PDGFRB, which are seen in other cancer types. ${ }^{17}$

Some nucleotide sequence variants identified by NGS can be readily labeled as pathogenic if they truncate the reading frame or disrupt gene structure; however, many of the variants identified are missense mutations whose significance is more difficult to interpret. In silico analysis algorithms for missense mutations, such as PolyPhen-2, can help guide oncologists in determining which mutations are likely pathogenic or benign. ${ }^{18}$ In silico algorithms use at least 1 of the following 4 classes of abnormalities to predict pathogenicity: comparison of physiochemical characteristics of wild-type and variant amino acids, evolutionary conservation at the location of mutation, the evolutionarily tolerated amino acid range at the mutation, or the protein's structural considerations. ${ }^{19}$ For many algorithms, evolutionary fitness serves as a surrogate for pathogenicity.

The in silico analysis algorithm used in this case was PolyPhen-2, which is one of the many algorithms available but has a free, user-friendly interface available online that can be used on personal computers. ${ }^{8}$ PolyPhen- 2 is a decision tree algorithm that combines protein structural attributes with a prebuilt sequence alignment that only includes mammalian species. The protein structural attributes are elucidated from crystal structures and include residues in active sites, binding sites, and alpha helices, and solvent accessibility. ${ }^{18,19}$ PolyPhen-2 then calculates a Position-Specific Independent Counts (PSIC) score that is the difference between the wild-type and mutant amino acid and converts the score into 1 of 3 categories: benign, possibly damaging, or probably damaging, as seen in Table 1. PolyPhen has been validated multiple times. ${ }^{18,20,21}$ In a recent study, Chan et $\mathrm{al}^{20}$ used curated locusspecific databases that covered 254 substitutions in 5 genes to reveal an overall predictive score of $75.0 \%$ when using PolyPhen. 
For our patient, in silico analysis predicted a benign change of ERBB2 compared with probably damaging mutations in KIT and PDGFR. When combined with preclinical studies indicating that $\mathrm{UC}$ is an angiogenic tumor overexpressing vascular endothelial growth factor (VEGF), we selected the targeted therapy pazopanib to treat our patient's heavily pretreated mUCRP. ${ }^{11}$ Pazopanib is an oral angiogenesis inhibitor that targets VEGF receptor, platelet-derived growth factor receptor, and KIT and is indicated for the treatment of metastatic renal cell carcinoma and soft tissue sarcoma. ${ }^{22}$ The results of 3 small clinical trials evaluating pazopanib in the treatment of metastatic UC are summarized in Table 2..$^{23}$ The contrasting results of the trials evaluating pazopanib alone do not provide a definitive answer on the utility of pazopanib in the treatment of metastatic UC. ${ }^{23,24}$ Interestingly, 4 out of the 7 PRs in the study by Necchi et $\mathrm{al}^{24}$ occurred in patients with UC of the upper urinary tract. PDGFRB is a receptor targeted by pazopanib, yet it was not a biomarker evaluated in the clinical trials performed. ${ }^{23,24}$ Genetic heterogeneity of heavily pretreated metastatic UC may explain the contrasting results seen in the clinical trials reported to date.

Pazopanib is not the only selective tyrosine kinase inhibitor (TKI) targeting the VEGF axis that has been used to date for metastatic UC. Gallagher et $\mathrm{al}^{26}$ used sunitinib, a TKI that inhibits VEGFR, PDGFR, KIT, FLT3, and RET, in a recent phase II study of 77 patients with metastatic UC, including 16 with mUCRP. A PR was seen in only 4 of the 77 patients. However, SD was seen in another 18 patients, indicating that sunitinib has clinical activity against metastatic
UC and that the VEGF axis is a viable target for novel therapies.

Another promising therapeutic class for the treatment of metastatic UC is anti-programmed death-ligand 1 (PD-L1) antibodies. ${ }^{27}$ Expression of PD-L1 enables cancer cells to elude immune surveillance and is thought to play a significant role in UC. A recent study has shown anti-PD-L1 immunotherapy to have promise in the treatment of metastatic UC, although a detailed discussion of this is outside the scope of this article. ${ }^{27}$

\section{Conclusions}

mUCRP is a rare disease with a poor prognosis due to a paucity of effective second-line therapies. Genomic platforms may yield clinically meaningful, individualized results that benefit patients facing such a challenging disease. Even though NGS can highlight potential targets, clinicians still face a challenge in deciphering which genomic alterations should be acted upon. Our case presents a unique use of in silico analysis with PolyPhen-2 to help oncologists determine which mutations should be targeted from WES results. We propose that in silico testing may be a useful, free, and easily accessible way to prioritize abnormalities found by NGS when clinical annotation is not definitive.

\section{Acknowledgments}

Author Contributions: MGM treated the patient and performed in silico analysis; MGM, AWH, and SG wrote the initial manuscript; MGM, AWH, DP, AV, HS, and SG completed all revisions.

\begin{tabular}{|c|c|c|c|}
\hline & Pili et $\mathrm{al}^{23}$ & Necchi et $\mathrm{al}^{24}$ & Gerullis et $\mathrm{al}^{25}$ \\
\hline Number of patients & 19 & 41 & 5 \\
\hline Chemotherapy used & Pazopanib, 800 mg/d & Pazopanib, $800 \mathrm{mg} / \mathrm{d}$ & $\begin{array}{l}\text { Pazopanib, } 200 \mathrm{mg}+ \\
\text { vinflunine, } 280 \mathrm{mg}\end{array}$ \\
\hline \multirow[t]{2}{*}{ Response rate (PR or CR) } & PR: 0 & PR: 7 & PR: 0 \\
\hline & CR: 0 & CR: 0 & CR: 0 \\
\hline $\begin{array}{l}\text { Adverse effects: number } \\
\text { and types }\end{array}$ & $\begin{array}{l}9 \text { grade } 3 \text { : anemia, } \\
\text { thrombocytopenia, } \\
\text { leukopenia, fatigue }\end{array}$ & $\begin{array}{l}\text { Grade } 3: \operatorname{HTN}(3), \\
\text { fatigue (2), GI and } \\
\text { vaginal fistulas (4) }\end{array}$ & $\begin{array}{l}\text { Trial discontinued at phase } \\
\text { I due to } 2 \text { of } 5 \text { patients with } \\
\text { dose-limiting toxicities }\end{array}$ \\
\hline
\end{tabular}

Abbreviations: CR, complete response; Gl, gastrointestinal; HTN, hypertension; PR, partial response. 
In Silico Analysis in Therapeutic Decisions

\section{References}

1. Raman JD, Messer J, Sielatycki JA, Hollenbeak CS. Incidence and survival of patients with carcinoma of the ureter and renal pelvis in the USA, 19732005. BJU Int 2011;107:1059-1064.

2. Gallagher DJ, Milowsky MI, Bajorin DF. Advanced bladder cancer: status of first-line chemotherapy and the search for active agents in the secondline setting. Cancer 2008;113:1284-1293.

3. Morrison CD, Liu P, Woloszynska-Read A, et al. Whole-genome sequencing identifies genomic heterogeneity at a nucleotide and chromosomal level in bladder cancer. Proc Natl Acad Sci U S A 2014;111:E672-681.

4. Ross JS, Wang K, Al-Rohil RN, et al. Advanced urothelial carcinoma: next-generation sequencing reveals diverse genomic alterations and targets of therapy. Mod Pathol 2014;27:271-280.

5. Chang F, Li MM. Clinical application of amplicon-based next-generation sequencing in cancer. Cancer Genet 2013;206:413-419.

6. Tse C, Shoemaker AR, Adickes J, et al. ABT-263: a potent and orally bioavailable Bcl-2 family inhibitor. Cancer Res 2008;68:3421-3428.

7. Wallace MD, Pfefferle AD, Shen L, et al. Comparative oncogenomics implicates the neurofibromin 1 gene (NF1) as a breast cancer driver. Genetics 2012;192:385-396.

8. PolyPhen-2: Prediction of Functional Effects of Human nsSNPs. Available at: http://genetics.bwh.harvard.edu/pph2/. Accessed September 9, 2015.

9. Ganjoo KN, Villalobos VM, Kamaya A, et al. A multicenter phase II study of pazopanib in patients with advanced gastrointestinal stromal tumors (GIST) following failure of at least imatinib and sunitinib. Ann Oncol 2014;25:236-240

10. Kwak C, Lee SE, Jeong IG, Ku JH. Adjuvant systemic chemotherapy in the treatment of patients with invasive transitional cell carcinoma of the upper urinary tract. Urology 2006;68:53-57.

11. Dasanu CA, Ong-Bacay A, Codreanu I. Newer developments in the therapeutics of the transitional cell carcinoma of renal pelvis. J Oncol Pharm Pract 2012;18:97-103.

12. Sternberg CN, Yagoda A, Scher HI, et al. Methotrexate, vinblastine, doxorubicin, and cisplatin for advanced transitional cell carcinoma of the urothelium. Efficacy and patterns of response and relapse. Cancer 1989;64:2448-2458.

13. Ross JS, Wang K, Gay L, et al. Comprehensive genomic profiling of carcinoma of unknown primary site: new routes to targeted therapies. JAMA Oncol 2015;1:40-49.
14. Bao R, Huang L, Andrade J, et al. Review of current methods, applications, and data management for the bioinformatics analysis of whole exome sequencing. Cancer Inform 2014;13(Suppl 2):67-82.

15. Yadav SS, Li J, Lavery HJ, et al. Next-generation sequencing technology in prostate cancer diagnosis, prognosis, and personalized treatment. Urol Oncol 2015;33:267.e1-13.

16. Precipio Diagnostics. SmartGen. Available at: http://www.precipiodx.com/ Smartgen.html\#sthash.u6OFvI6n.dpbs. Accessed September 9, 2015.

17. Comprehensive molecular characterization of urothelial bladder carcinoma. Nature 2014;507:315-322

18. Adzhubei IA, Schmidt S, Peshkin L, et al. A method and server for predicting damaging missense mutations. Nat Methods 2010;7:248-249.

19. Tavtigian SV, Greenblatt MS, Lesueur F, Byrnes GB. In silico analysis of missense substitutions using sequence-alignment based methods. Hum Mutat 2008;29:1327-1336.

20. Chan PA, Duraisamy S, Miller PJ, et al. Interpreting missense variants: comparing computational methods in human disease genes CDKN2A, MLH1, MSH2, MECP2, and tyrosinase (TYR). Human Mutat 2007;28:683-693.

21. Chao EC, Velasquez JL, Witherspoon MS, et al. Accurate classification of MLH1/MSH2 missense variants with multivariate analysis of protein polymorphisms-mismatch repair (MAPP-MMR). Human Mutat 2008;29:852-860.

22. Sternberg CN, Davis ID, Mardiak J, et al. Pazopanib in locally advanced or metastatic renal cell carcinoma: results of a randomized phase III trial. J Clin Oncol 2010;28:1061-1068.

23. Pili R, Qin R, Flynn PJ, et al. A phase II safety and efficacy study of the vascular endothelial growth factor receptor tyrosine kinase inhibitor pazopanib in patients with metastatic urothelial cancer. Clin Genitourin Cancer 2013;11:477-483.

24. Necchi A, Mariani L, Zaffaroni N, et al. Pazopanib in advanced and platinum-resistant urothelial cancer: an open-label, single group, phase 2 trial. Lancet Oncol 2012;13:810-816.

25. Gerullis H, Eimer C, Ecke TH, et al. Combined treatment with pazopanib and vinflunine in patients with advanced urothelial carcinoma refractory after first-line therapy. Anticancer Drugs 2013;24:422-425.

26. Gallagher DJ, Milowsky MI, Gerst SR, et al. Phase II study of sunitinib in patients with metastatic urothelial cancer. J Clin Oncol 2010;28:13731379.

27. Powles T, Eder JP, Fine GD, et al. MPDL3280A (anti-PD-L1) treatment leads to clinical activity in metastatic bladder cancer. Nature 2014;515:558-562. 